\title{
Pulse Distortion in Saturated Fiber Optical Parametric Chirped Pulse Amplification
}

\author{
Lali-Dastjerdi, Zohreh; Da Ros, Francesco; Rottwitt, Karsten; Galili, Michael; Peucheret, Christophe
}

Published in:

CLEO Technical Digest

Publication date:

2012

Document Version

Publisher's PDF, also known as Version of record

Link back to DTU Orbit

Citation (APA):

Lali-Dastjerdi, Z., Da Ros, F., Rottwitt, K., Galili, M., \& Peucheret, C. (2012). Pulse Distortion in Saturated Fiber Optical Parametric Chirped Pulse Amplification. In CLEO Technical Digest (pp. JW2A.82). Optical Society of America.

\section{General rights}

Copyright and moral rights for the publications made accessible in the public portal are retained by the authors and/or other copyright owners and it is a condition of accessing publications that users recognise and abide by the legal requirements associated with these rights.

- Users may download and print one copy of any publication from the public portal for the purpose of private study or research.

- You may not further distribute the material or use it for any profit-making activity or commercial gain

- You may freely distribute the URL identifying the publication in the public portal 


\title{
Pulse Distortion in Saturated Fiber Optical Parametric Chirped Pulse Amplification
}

\author{
Zohreh Lali-Dastjerdi,* Francesco Da Ros, Karsten Rottwitt, Michael Galili, and Christophe Peucheret \\ DTU Fotonik, Department of Photonics Engineering, Technical University of Denmark, DK-2800 Kgs. Lyngby, Denmark \\ *Corresponding author: zoda@fotonik.dtu.dk
}

\begin{abstract}
Fiber optical parametric chirped pulse amplification is experimentally compared for different chirped pulses in the picosecond regime. The amplified chirped pulses show distortion appearing as pedestals after recompression when the amplifier is operated in saturation.

OCIS codes: (190.4410) Nonlinear optics, parametric processes; (190.4970) Parametric oscillators and amplifiers
\end{abstract}

\section{Introduction}

Fiber optical parametric chirped pulse amplification (FOPCPA) has recently been the focus of studies as a promising amplification scheme for pico- and femto-second pulses in optical fibers [1-3]. Since parametric amplification is an ultrafast process with gain strongly dependent on the input signal power [4], the output of an FOPCPA depends on the temporal profile of the input chirped signal, an effect that is revealed in the amplified signal after recompression. The amplified signal distortion can be investigated operating in the nonlinear gain regime to highlight the effect of the parametric gain response. Recent numerical studies have reported the appearance of pulse distortion in the form of pedestals around the recompressed signal in the nonlinear gain regime [5-6]. In this work, we have experimentally compared FOPCPA for differently chirped pulses under transition from linear to saturated gain regime and investigated the signal distortion after recompression. We experimentally show, for the first time to the best of our knowledge, that chirped pulse distortion in FOPCPA during amplification cannot be recovered by recompression.

\section{Results and discussion}

The experimental set up is shown in Fig. 1. The pump signal is a continuous wave at $1557.5 \mathrm{~nm}$ that is phase modulated using a $10 \mathrm{~Gb} / \mathrm{s} 2^{7}-1$ pseudorandom binary sequence to suppress stimulated Brillouin scattering, amplified by an erbium-doped fiber amplifier (EDFA) up to $28.5 \mathrm{dBm}$ and filtered by a 1-nm bandwidth optical band pass filter (OBPF). The chirped signal pulses are generated through a tunable laser followed by an intensity modulator (IM) and a phase modulator (PM) synchronously driven by sinusoidal electrical signals at $40 \mathrm{GHz}$. The pulses are then amplified, filtered by 5 -nm bandwidth OBPF and the power at the input to the amplifier is controlled by a variable optical attenuator (VOA). A 10/90\% coupler combines the pump and signal into a highly nonlinear fiber (HNLF) characterized by the parameters reported in the caption of Fig. 1. The amplified signal is finally filtered by a 5-nm bandwidth OBPF. The output of the PM is a positively chirped (PC) pulse, with 5.4-ps pulse width as measured by an autocorrelator, which calculates the FWHM of the pulse by deconvoluting the autocorrelation under the assumption of a $\operatorname{sech}^{2}$ pulse shape. Compressing the output of the PM by $250 \mathrm{~m}$ of standard single mode fiber (SMF) will result in negatively chirped pulses (hereafter referred to as pre-compressed pulses) with 2.9 ps width, as indicated in the inset of Fig. 2

The dynamics of amplification of chirped pulses in FOPCPA has been investigated for the generated broad PC and narrow pre-compressed pulses. Furthermore, the broad PC pulses have been post-compressed after filtering the amplified signal (using the same amount of SMF as for pre-compression) in order to evaluate the quality of the amplified signal when moving from the linear to the nonlinear gain regime. The signal gain at $1547 \mathrm{~nm}$ (which corresponds to the phase matched wavelength, resulting in maximum small signal gain) has been characterized as a function of the signal input power for the pre- and post-compressed pulses, as shown in Fig. 2.
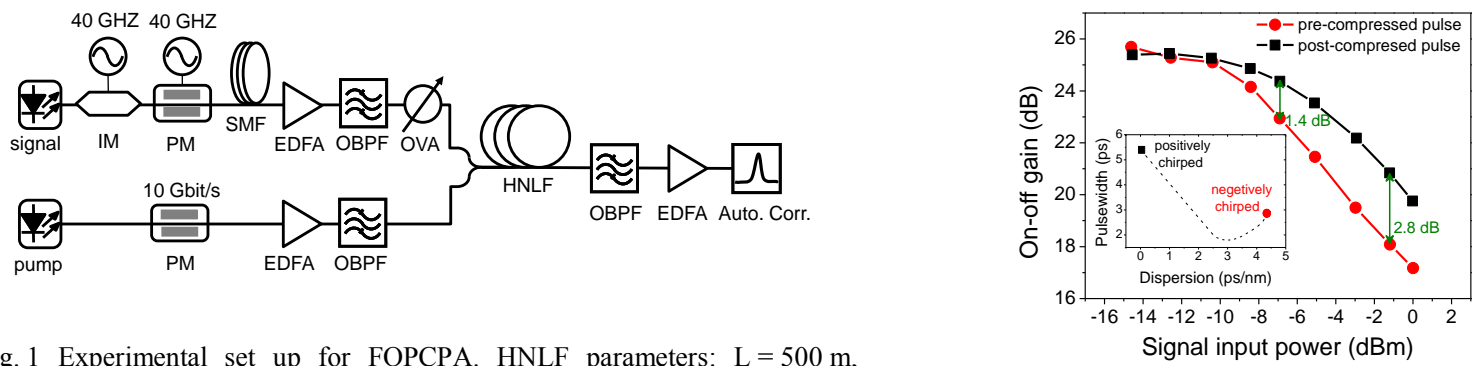

Fig. 1 Experimental set up for FOPCPA. HNLF parameters: $\mathrm{L}=500 \mathrm{~m}$, $\gamma=10.7 \mathrm{~W}^{-1} \mathrm{~km}^{-1}, \mathrm{~S}=0.0185 \mathrm{ps} /\left(\mathrm{nm}^{2} \cdot \mathrm{km}\right), \lambda_{0}=1550.4 \mathrm{~nm}, \alpha=0.7 \mathrm{~dB} / \mathrm{km}$.

Fig. 2 On-off gain as a function of signal input power. 
In the linear gain regime, both signals have the same gain, but by increasing the signal input power, the gain saturates faster for the shorter pre-compressed pulses than for the broader post-compressed pulses. In fact, the gain saturation depends on the signal peak power as the parametric gain responds fast to the input signal time variations. The gain difference at $\mathrm{Ps}=-7 \mathrm{dBm}$ is $1.4 \mathrm{~dB}$ and it even increases to $2.8 \mathrm{~dB}$ for $\mathrm{Ps}=-1.2 \mathrm{dBm}$. The pulse broadening evolution under saturation for three situations (PC, pre- and post-compressed pulses) is illustrated in Fig. 3. The measurements show that the pulses are temporally broadened when operating in gain saturation. The broadening rate, however, is different for different amounts of chirp. Comparing the broad PC and narrow precompressed pulses shows that the broadening is faster for the latter. The pulse shapes in the three cases at different saturation levels are shown in Fig. 4. The shape of the narrow pre-compressed pulse shows strong pedestals in deep saturation (middle plot).

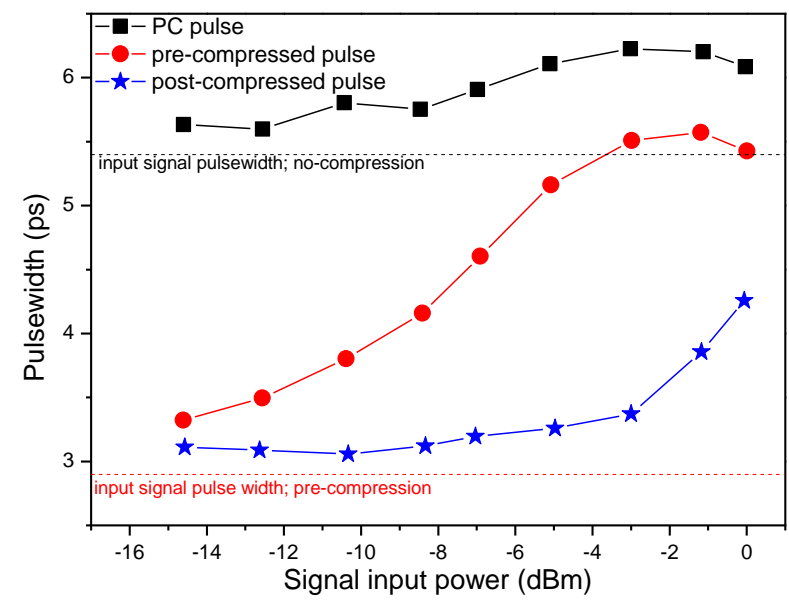

Fig. 3 Pulse width as a function of the signal input power for broad PC, narrow NC and post-compressed pulses.
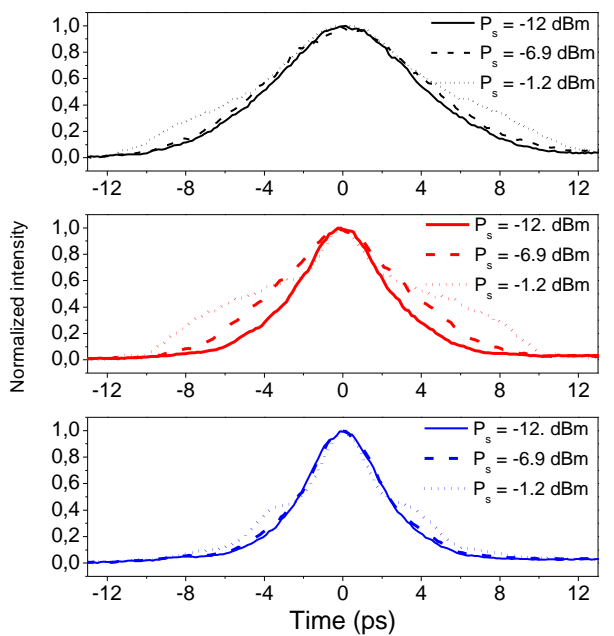

Fig. 4 Autocorrelation trace for the PC (top), pre-compressed (middle) and post-compressed pulses (bottom) under different saturation levels.

Furthermore, the comparison between pre-and post compression in the linear regime shows the same pulse shape, as shown in Fig. 4 (solid curves in the middle and bottom plots). The pulse broadening is mild in the case of post-compression and the pulse shape can be maintained for higher signal input powers. This can be related to the lower nonlinear interaction during amplification for temporally broad pulses. The amplified pulse preserves its shape and width up to $\mathrm{P}_{s}=-3 \mathrm{~dB}$ (blue stared curve in Fig. 3), i.e. when the gain is saturated by more than $3 \mathrm{~dB}$. For higher saturation levels, e.g. $\mathrm{P}_{\mathrm{s}}=-1.2 \mathrm{dBm}$ in Fig. 4, the PC signal demonstrates that the pedestals emerging around the amplified signal (top plot) cannot be removed by post-compression (bottom plot). The experiments thus confirm the results shown only numerically in $[5,6]$.

The effect of the signal dispersion is here limited as the signal is located in the weak normal-dispersion region of the HNLF ( $3.4 \mathrm{~nm}$ below the zero-dispersion wavelength). Our numerical studies, which are not presented here, have however disclosed that the pulse quality can also be affected by the signal dispersion, depending on the chirp of the signal at the input of the amplifier. These effects are currently under further investigation.

In conclusion, FOPCPA has been experimentally investigated for different chirped signals in the linear and nonlinear gain regimes. The broadly chirped signals have higher saturation powers, which result in reduced pulse distortion for higher signal input powers. The amplified chirped signals in highly saturated amplifiers show excess distortion under the form of pedestals around the amplified signal after recompression.

\section{References}

[1] M. Hanna, F. Druon, and P. Georges, "Fiber optical parametric chirped-pulse amplification in the femtosecond regime," Opt. Express 14, 2783-2790 (2006).

[2] Y. Zhou, Q. Li, K. Cheung, S. Yang, P. Chui, and K. Wong, "All-fiber-based ultrashort pulse generation and chirped pulse amplification through parametric processes," IEEE Photon. Technol. Lett. 22, 1330-1332 (2010).

[3] C. Caucheteur, D. Bigourd, E. Hugonnot, P. Szriftgiser, A. Kudlinski, M. Gonzalez-Herraez and A. Mussot, "Experimental demonstration of optical parametric chirped pulse amplification in optical fiber," Opt. Lett. 35, 1786- 788 (2010).

[4] M. E. Marhic, Fiber optical parametric amplifiers, oscillators and related devices (Cambridge University Press, 2008).

[5] D. Bigourd, L. Lago, A. Ludlinski, E. Hugonnot and A. Mussot, "Dynamics of fiber optical parametric chirped pulse amplifiers," J. Opt. Soc. Am. B 28, 2848-2854 (2011).

[6] C. Manzoni, J. Moses, F. X. Kärtner and G. Cerullo, “Excess quantum noise in optical parametric chirped-pulse amplification,” Opt. Express 19, 8357-8366 (2011) 